\title{
Intraosseous Mandibular Capillary Hemangioma Most Likely of Traumatic Origin
}

\section{Neji Omar ${ }^{1}$, Moussaoui Eya ${ }^{1,2 *}$, Belkacem Raouâa ${ }^{1,2}$, Sriha Badreddine $^{3}$, Oualha Lamia ${ }^{1}$ and Douki Nabiha ${ }^{1,2}$}

${ }^{1}$ Department of Dental Medicine, EPS Sahloul, Sousse, Tunisia

${ }^{2}$ Faculty of Dental Medicine, Oral Health and Oro-Facial Rehabilitation Laboratory

Research (LR12ES11), University of Monastir, 5019 Monastir, Tunisia

${ }^{3}$ Cytology and Pathological Anatomy Department, Farhat Hached Hospital, University of Sousse, Sousse, Tunisia

*Corresponding Author: Moussaoui Eya, Department of Dental Medicine, EPS

Sahloul, Sousse, Tunisia.
Received: September 13, 2021

Published: October 18, 2021

(C) All rights are reserved by Moussaoui Eya., et al.

\begin{abstract}
Intraosseous Hemangiomas account for approximately $0.5 \%$ to $1 \%$ of all intraosseous tumors. They are frequently located in the vertebral skeleton than the facial bones.

They usually occur in the cavernous type. The capillary type is rarer.

It is usually asymptomatic and most often discovered incidentally on plain radiographs.

The pathogenesis of intraosseous hemangioma remains unknown. It could be congenital or due to previous trauma.

Treatment of hemangiomas varies depending on the lesion's extension and dimension. It consists basically on surgical excision with or without preoperative embolization.

In this paper, we report a case of a 51-year-old woman with intraosseous mandibular capillary hemangioma, located on the tip of the mandibular ridge evocating a traumatic origin, probably due to the extraction of the 46 . It was treated by a simple resection without postoperative complications.
\end{abstract}

Keywords: Central Hemangioma; Intraosseous Hemangioma; Capillary Hemangioma; Traumatic Origin; Embolization; Osteolytic Lesion; Polka-dot Appearance; Angiography

\section{Introduction}

Intraosseous hemangioma, also known as central hemangioma, is a benign vascular tumor, accounting for only $0.5 \%$ to $1 \%$ of all intraosseous tumors [1].

It is rare in the facial bone and exceptional in the maxillaries [2].

The origin of central hemangioma is still debatable. Some authors believe that it is a true neoplasm while others consider it as a hamartomatous lesion [3].
Its Pathogenesis remains unknown, being either congenital or consecutive to a previous trauma [4].

Histologically, capillary and cavernous hemangiomas are distinguished [5].

In this paper, we report a case of a 51-year-old woman with a capillary hemangioma, located on the tip of the mandibular ridge evocating a traumatic origin, probably due to the extraction of the 46. 


\section{Case Report}

A 51-year-old woman with a medical history of high blood pressure was referred to our department by her general dental practitioner for the evaluation of a swelling located in the extraction site of the 46, appearing one month before. The tooth was removed 10 years earlier.

Intraoral examination showed a firm swelling measuring $2 \mathrm{~cm}$ in diameter with a normal mucosa located in the right mandibular ridge. However, a distolingual bluish spot was noticed. No other associated symptoms were present (Figure 1).

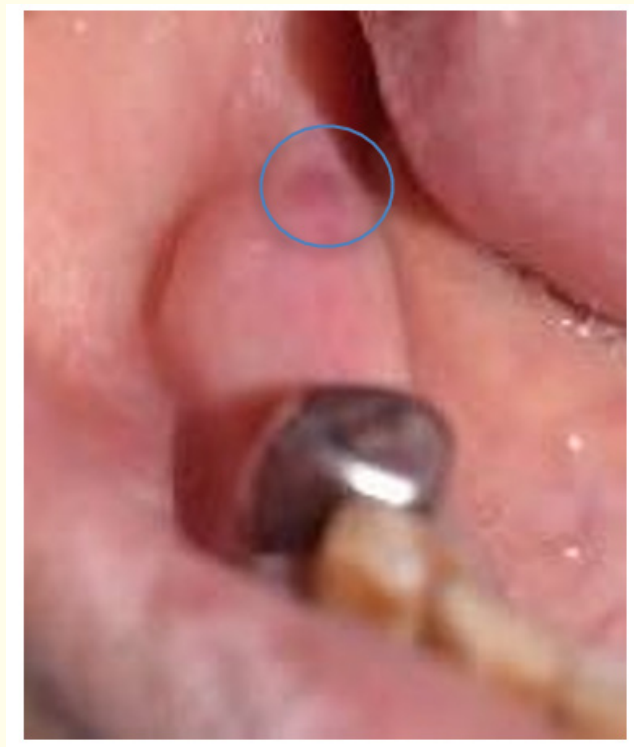

Figure 1: Intraoral examination: a firm swelling covered by normal mucosa. note the distolingual bluish spot.

Dental panoramic radiograph revealed an osteolytic lesion with a well-defined radiolucent image of approximately $8 \mathrm{~mm}$ in diameter located on the tip of the mandibular ridge, presenting a distal discontinuity of approximately $2 \mathrm{~mm}$, and corresponding to the bluish spot observed in the clinical examination (Figure 2).

Differential diagnosis included residual bone cyst and bone capillary hemangioma. The treatment consisted in the lesion enucleation.

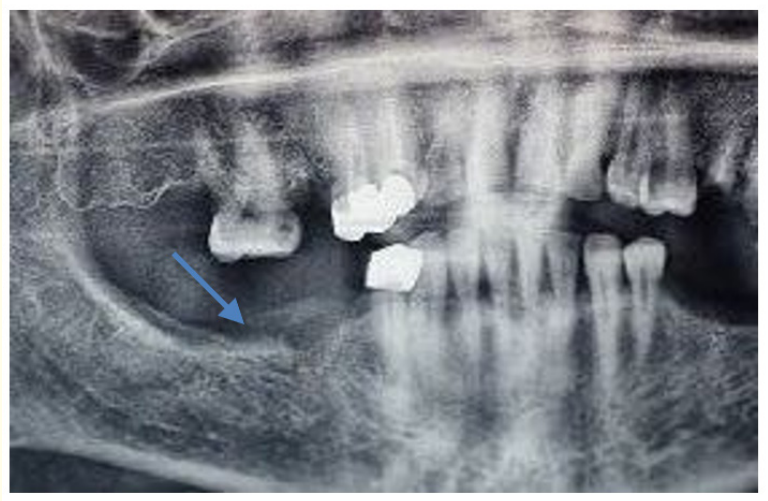

Figure 2: Panoramic radiographic: an osteolytic lesion with a well-defined radiolucent image, presenting a distal discontinuity of $2 \mathrm{~mm}$ (blue arrow).

During the surgery, excessive bleeding was observed on the lesion site.

Histological examination was non specific and revealed a firosis, including blood capillaries and inflammatory cells (Figure 3).

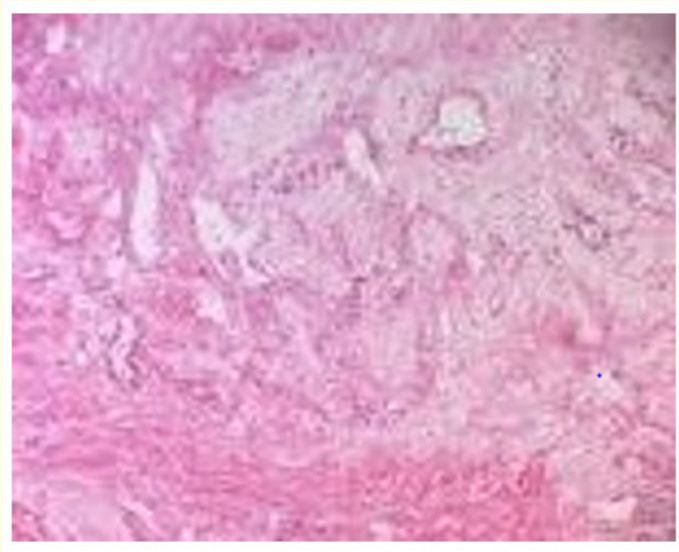

Figure 3: Histological examination showed a firosis, including blood capillaries and inflammatory cells.

The histological diagnosis was not convincing since the patient returned two weeks later with a budding appearance of the excisi- 
on site and absence of tissue healing associated with an ulcerative surface (Figure 4). This clinical feature was very consistent with pyogenic granuloma.

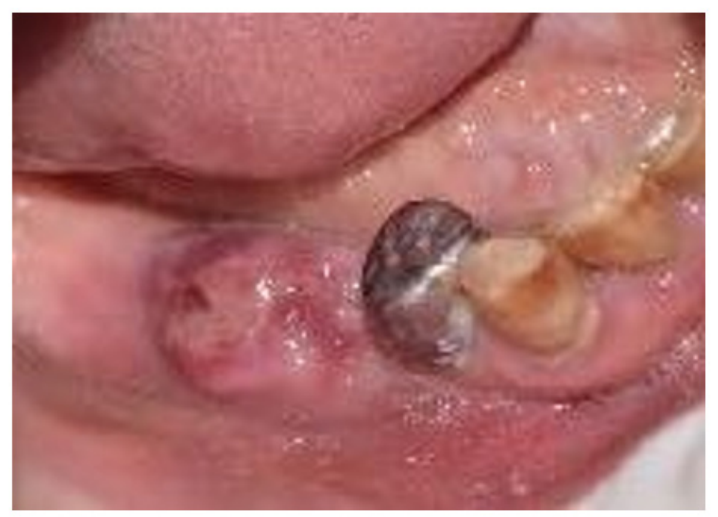

Figure 4: Absence of tissue healing associated with an ulcerative surface.

Amoxicillin was prescribed for 7 days. After 5 weeks, the lesion kept the same diameter but with bluish and reddish appearance (Figure 5). Palpation showed a spongy mass.

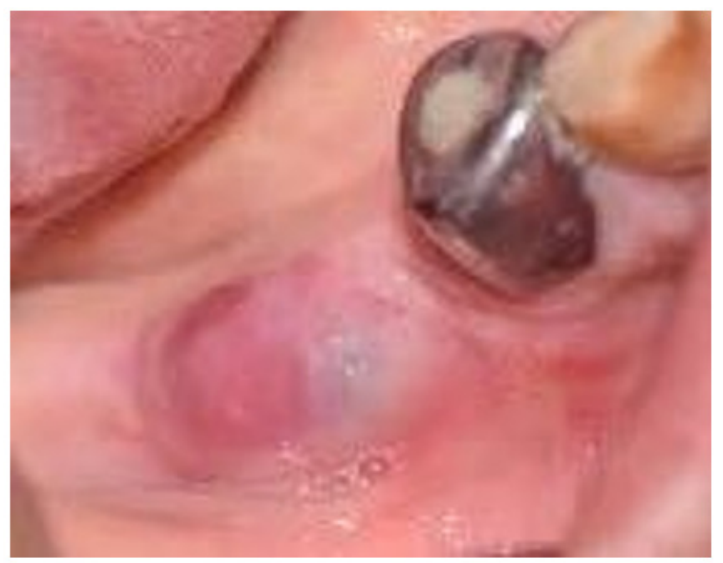

Figure 5: Bluish and reddish appearance of the swelling.

A second resection was performed. During the surgery, bleeding was noticed and it was controlled using local compression associa- ted with the use of tranexamic acid. The surgical specimen of $5 \mathrm{~mm}$ in diameter was encapsulated and it had a soft consistency and a bluish appearance (Figure 6).

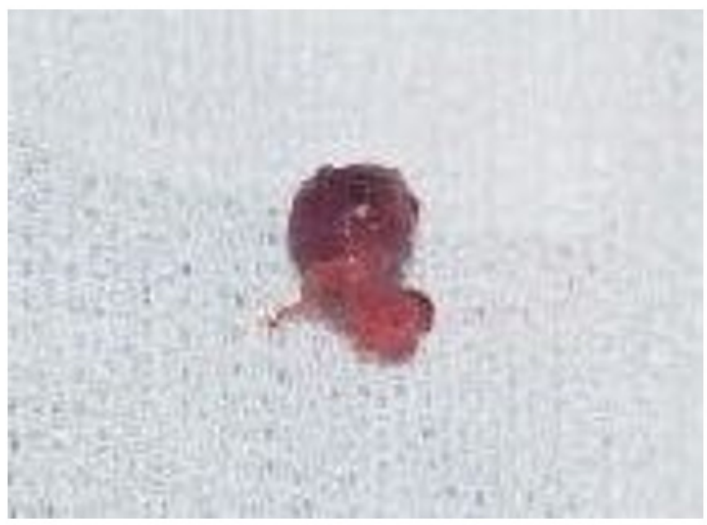

Figure 6: The surgical specimen was encapsulated with a bluish appearance.

CT scan was not performed because of the patient's financial conditions and because we have an idea about the low blood flow of the lesion that was noticed during the first surgery.

Histological examination showed veinal structures with a dilated lights, which was largely thrombosed. The walls were thickened and fibrous (Figure 7). Diagnosis of intraosseous capillary hemangioma with venous thrombosis was finally confirmed. After the 3,6 , and 12 months of medical monitoring, a satisfying healing with no recurrence was noticed (Figure 8 a,b).

\section{Discussion}

According to the World Health Organization, hemangiomas are benign vasoformative neoplasms [1].

Intraosseous Hemangiomas account for approximately $0.5 \%$ to $1 \%$ of all intraosseous tumors [1]. They are most commonly found in females than in males [6]. They are frequently located in the vertebral skeleton than the facial bones. In the head, the most common site is the parietal bone, followed by the mandible, then the molar and zygomatic regions $[6,7]$. 


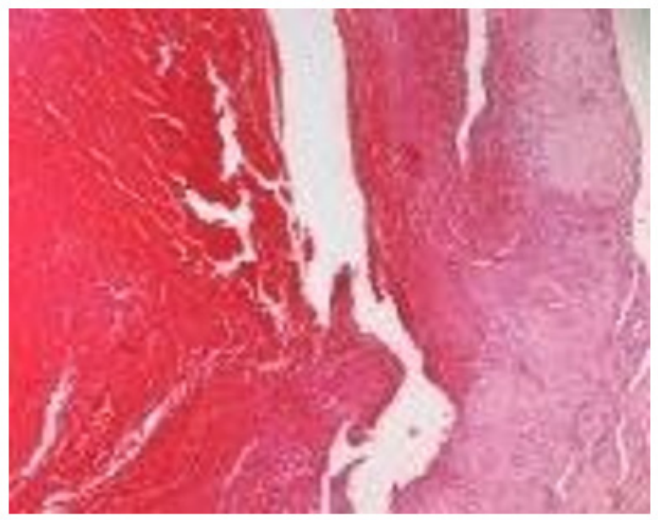

Figure 7: Histological examination: veinal structures with a dilated lights, which was largely thrombosed.
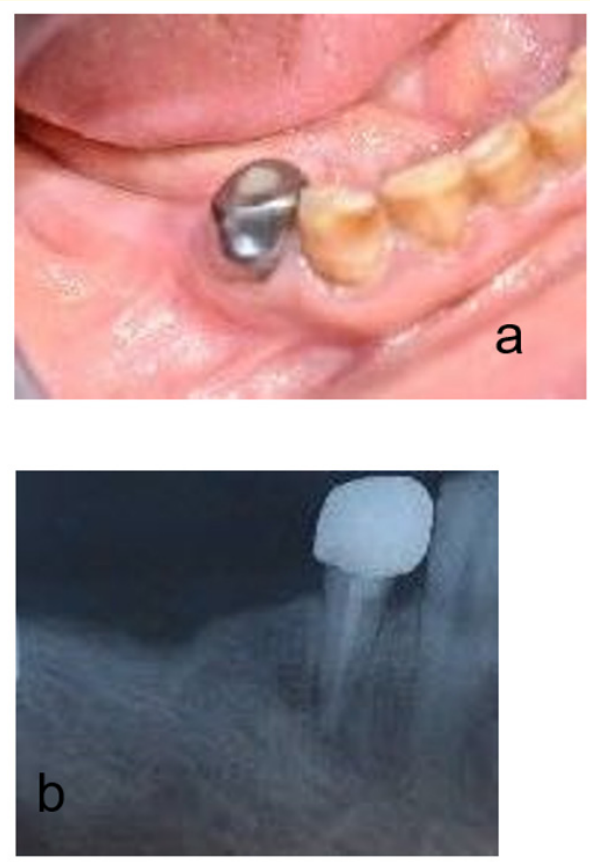

Figure 8: Control after 12 months.

a. Intraoral healing

b. Periapical radiograph: total regression of the radiolucency.
Histologically, they are classified into capillary, cavernous, mixed, and sclerosing types [2,7].

Intraosseous hemangiomas usually occur in the cavernous type. They are usually asymptomatic and most often discovered incidentally on plain radiographs [2]. The capillary type is rarer.

The pathogenesis of intraosseous hemangioma remains unknown. It could be congenital but this theory has not been proved yet. A previous trauma could be an important etiology to be considered [4]. In fact, trauma is believed to be a common cause of primary intraosseous hemangiomas [6].

In this case, a traumatic origin was considered for many reasons: history of extraction of the 46 and the location of the angioma on the tip of the crest.

The clinical presentations of intraosseous hemangioma are variable and independent from the histological type. Several patients report an experience of pain and swelling. Paresthesia of the mental region and lips can be seen. A sense of pulsation at the involved region in case of high-pressure hemangiomas is also reported $[1-3,8,9]$. Intraosseous capillary hemangiomas are exceptional and only few cases have been reported. Perugini., et al. reported two cases of capillary hemangioma on the mandible [10]. Mutlu., et al. reported a case of intraosseous

capillary hemangioma in a newborn without significant extra-oral findings [11].

Intra-orally, capillary hemangiomas are characterized by a color range between red and blue depending on the profundity of the lesion. The surface is smooth and shiny with loss of stippling of the involved gingiva. On palpation, the swelling is bony hard and non-tender. No pulsation or bruit is noted. The lesion can bleed on provocation and a mobility of the involved teeth can be observed. The dentist must be aware of the lesion as severe bleeding may be encountered during tooth extraction or biopsy [1-3,8,9].

In our case, the bluish appearance and the spongy mass noticed in the second surgery were due to the elimination of the lesion's upper bone during the first surgery. This can be explained by the fact that hematic content of the lesion was visible by transparency through the gingiva. 
The radiological appearance of central capillary hemangiomas is not pathognomonic. In standard radiographs, alterations in the trabecular pattern varying from thin to thicker, or a well-defined radiolucent image of varying size, usually located deeply in the bone, can be found $[1-3,9]$.

Dental panoramic radiograph in the present case revealed the presence of a well- defined radiolucent image of $8 \mathrm{~mm}$ in diameter.

CT-scan allows clear visualization of the cortical involvement and it is also useful for determining the extension of hemangioma and its relationship with the surrounding soft tissues [3].

The polka-dot appearance is the classical CT scan feature [1$3,9]$.

Angiography can be used as a diagnostic tool to demonstrate the presence of a vascular lesion, to differentiate between arteriovenous fistula and central hemangioma, and to visualize the feeding artery of the vascular lesion [1,3].

In the present case, angiography was not performed because of the small size of the lesion and the patient's financial conditions.

The histological examination of intraosseous capillary hemangioma is characterized by the presence of numerous small-sized vessels that are consistent with capillary vessels, especially in the proliferative phase, the presence of a single layer of endothelial cells and lacked muscular coat, the presence of few inflammatory cells, and the absence of inter-cellular edema in the connective tissue stroma $[1-3,8,9]$.

In this case, the first biopsy revealed the presence of a focus of fibers, including blood capillaries, and the presence of inflammatory cells. The second biopsy revealed a venular structure with dilated section, largely thrombosed with a thickened and fibrous wall. These two results allowed for the diagnosis of a histological appearance of intraosseous capillary angioma with venous thrombosis.

Differential diagnosis includes ameloblastoma and mixoma, especially for Cavernous hemangiomas and capillary ones with a honeycombed appearance. It also includes cystic lesions, such as odontogenic cysts and aneurismatic bone cysts, as well as fibrous lesions, especially osseous dysplasias in their initial phase of development $[1-3,8,9]$.
In our case, due to the lesion's location and aspect, the main differential diagnosis was residual cyst.

Treatment of hemangiomas varies depending on the lesion's extension and dimension [1,2,5,12]. Surgical excision with or without preoperative embolization is most frequently indicated $[1,2,5,8,12]$. The use of sclerosing agents is an effective, safe, and inexpensive alternative solution $[1,2,5,8,12]$.

Injected steroid therapy acts to reduce blood flow in the lesion. In case of resistance, the use of interferon alpha can be indicated $[1,2,5,12]$.

Laser therapy is widely used as a therapeutic modality for Hemangiomas. It provides a low degree of bleeding during the excision of the lesion $[1,2,5,8,12]$.

In the current case, the lesion was surgically treated with simple curettage and bleeding was easily managed by the use of tranexamic acid.

After surgery, lesions rarely recur. Colin Bacron., et al. reported a period of 8 years without recurrence since the first resection [13].

Recurrence can be avoided by respecting a margin of safety [4].

In this reported case, no recurrence was observed until one year following resection.

\section{Conclusion}

Due to the rarity of intraosseous capillary hemangiomas, a gradually approach toward a definite diagnosis is required by excluding other similar bony lesions. the gold standard for a correct diagnosis is the association of histological examination, CT scan and angiography.

Treatment modality should be based on the patient's age, clinical features and lesion's extension.

\section{Bibliography}

1. Guillermo Gómez Oliveira., et al. "Intraosseous mandibular hemangioma. A case report and review of the literature". Medicina Oral, Patologia Oral, Cirugia Bucal 13.8 (2008): E496-498.

2. Dereci O., et al. "Unusual intraosseous capillary hemangioma of the mandible". European Journal of Dentistry 9 (2015): 438441. 
3. Debasmita Mitra., et al. "Central capillary hemangioma of the maxilla: Case report and a review of the literature". The Saudi Journal for Dental Research (2015): 2352-0035

4. Alejandra Arévalo Sáenz., et al. "Intraosseous cavernous hemangioma: presentation of a clinical case". Egyptian Journal of Neurosurgery 33 (2018): 22.

5. Mustafa Jamel Abdullah., et al. "Hemangioma of the Buccal Mucosa: A Case Report and Review of the Literature Scholars". Journal of Dental Sciences 3.8 (2016): 228-230.

6. David B Powers., et al. "Intraosseous Hemangioma: Case Report and Literature Review". Craniomaxillofacial Trauma Reconstruction 10 (2017): 1-10.

7. Mehmet Mutlu., et al. "Intraosseous noninvoluting congenital hemangioma of the mandible in a neonate". The Turkish Journal of Pediatrics 51 (2009): 507-509.

8. Dhiman NK., et al. "Central cavernous hemangioma of mandible: Case report and review of literature". National Journal of Maxillofacial Surgery 6 (2015): 209-213.

9. Nidhi Sinha., et al. Journal of Dentistry 3.1 (2016).

10. Maurizio Perugini., et al. "Intraosseous Hemangioma of the Maxillofacial District: Clinical Analysis and Surgical Treatment in 10 Consecutive Patients". The Journal of Craniofacial Surgery 5.6 (2004).

11. Mutlu M., et al. "Intraosseous non involuting congenital hemangioma of the mandible in a neonate". Turkish Journal of Pediatrics 51 (2009): 507-509.

12. Walessa Brasil da Silva., et al. "Oral capillary hemangioma: A clinical protocol of diagnosis and treatment in adults". Oral Maxillofacial Surgery 18 (2014): 431-437.

13. Colin Bacorn and Lily Koo Lin. Case Reports in Ophthalmological Medicine (2020).

Volume 5 Issue 11 November 2021

(C) All rights are reserved by Moussaoui Eya., et al. 